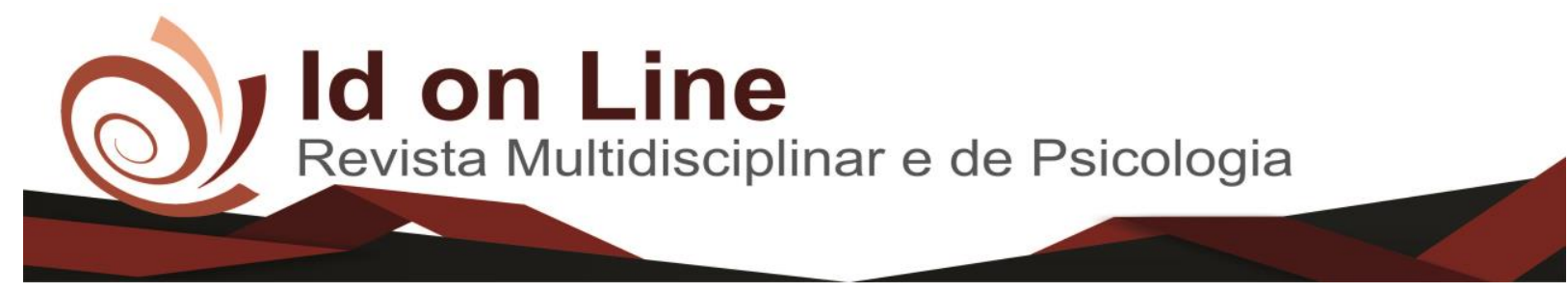

Artigo

\title{
Análise de Prescrições Médicas de medicamentos regulados pela Portaria federal 344/1998, dispensados em uma drogaria no interior da Bahia
}

\author{
Ana Katherine De Souza Debastiani ${ }^{1}$; Jaqueline Ferraz Rodrigues Coqueiro ${ }^{2}$.
}

Resumo: O avanço da ciência e a inovação tecnológica em medicamentos têm aumentado a expectativa de vida das pessoas demandando assim novos medicamentos. Numa drogaria, o profissional farmacêutico, é o responsável por todas as atividades inerentes a dispensação dos medicamentos e é necessário que ele esteja atento a todas as informações contidas na prescrição, para que o paciente siga as instruções e orientações do seu tratamento. Este estudo foi elaborado com o intuito de observar os aspectos legais relacionados à receita médica, a começar pela forma em que foi prescrita, de forma clara até a sua dispensação pelo farmacêutico na parte final do processo da aquisição do medicamento. Esta pesquisa trata-se de um estudo exploratório descritivo quantitativo, foi realizada em uma drogaria, localizada na cidade de Poções- BA. As análises foram feitas com base nos parâmetros estabelecidos pela Portaria Federal 344 de 12 de maio de 1998, através de um formulário de avaliação elaborado.

Palavras-chave: Prescrições. Psicotrópicos. SNGPC

\section{Medical Prescriptions analysis of drugs regulated by the federal Ordinance 344/1998, dispensed in a drugstore in Bahia}

\begin{abstract}
The advancement of science and technological innovation in medicines have increased the life expectancy of people, thus demanding new medicines. In a pharmacy, the pharmacist is the responsible for all inherent activities to the dispensation of medicines and this professional must be alert to all the information contained in the prescription, so the patient could follow the instructions and orientation of his treatment. This study was designed to observe the legal aspects related to medical prescription, starting from the way it was prescribed, clearly until dispensed by the pharmacist in the final part of the medication acquisition process. This research is an exploratory descriptive quantitative study, which was carried out in a drugstore, located in the city of Poções - BA. The analysis will be made based on the parameters established by Brazilian Federal Decree 344 of May 12, 1998, through an elaborated evaluation form.
\end{abstract}

Keywords: Prescriptions. Psychotropic drugs.SNGPC

${ }^{1}$ Graduanda em Farmácia Generalista pela Faculdade Independente do Nordeste.

${ }^{2}$ Farmacêutica Mestre em Planejamento Ambiental pela Universidade Católica do Salvador, UCSAL. Especialista em Saúde Pública com complementação em Magistério Superior, especialista em Saúde Mental pela Universidade Federal do Rio de Janeiro e em MBA em Auditoria em Serviços de Saúde; Docente do curso de Farmácia na Faculdade Independente do Nordeste (FAINOR). End. Para correspondência: Rua Benitto Schettini,s/n - Centro - Poções/BA. (77) 98115 1020. E-mail: katherine_debastiani@hotmail.com

118 Id on Line Rev. Mult. Psic. V.11, N. 39. 2017 - ISSN 1981-1179 Edição eletrônica em http://idonline.emnuvens.com.br/id 


\section{Introdução}

O grande avanço tecnológico das indústrias farmacêuticas aconteceu logo após a Segunda Guerra Mundial, com o desenvolvimento do comércio farmacêutico o que permitiu a ampliação da pesquisa e da produção de medicamentos e da oferta no mercado (MOREIRA, 2010).

O avanço da ciência e a inovação tecnológica em medicamentos têm aumentado a expectativa de vida das pessoas demandando assim novos medicamentos. (BRASIL, 2014).

De acordo com Albuquerque (2009) medicamentos são produtos farmacêuticos que tem como finalidade profilática, curativa, paliativa ou para fins de diagnostico. Um tratamento médico realizado através do uso de medicamentos, prescritos de acordo com o quadro clínico de uma determinada patologia, que visa proporcionar uma melhora à qualidade de vida dos pacientes.

O medicamento a ser consumido pelo paciente é definido em uma prescrição médica, com a respectiva dosagem e duração de tratamento é prescrito. Em geral, esse ato é expresso mediante a elaboração de uma receita médica (ALBUQUERQUE, 2009, p. 116). Para que ocorra um tratamento eficaz é fundamental compreender o que foi prescrito pelo médico para que não ocorram erros de medicação por parte do paciente ou dos profissionais de saúde.

Numa drogaria, o profissional farmacêutico, é o responsável por todas as atividades inerentes a dispensação dos medicamentos e é necessário que ele esteja atento a todas as informações contidas na prescrição, para que o paciente siga as instruções e orientações do seu tratamento.

Pela lei Federal 5991 de 1973 a drogaria é um estabelecimento de dispensação e de comércio de drogas, medicamentos, insumos farmacêuticos e correlatos em suas embalagens originais.

Os medicamentos considerados como de controle especial, são regulados pela Portaria Federal de no $344 / 98$, que é uma norma técnica onde normatiza o comércio destes medicamentos e apresenta também em anexo as listas com nomes das substâncias dos medicamentos controlados. De acordo com o Art. 35 da Portaria Federal de $n^{\circ} 344 / 98$, diz que a notificação de receita é o documento que acompanhado de receita autoriza a dispensação de medicamentos a base de substâncias constantes das listas "A1" e "A2" (entorpecentes), "A3", "B1" e "B2" (psicotrópicas), “C2” (retinóicas para uso sistêmico) e "C3" (imunossupressoras). 
Os medicamentos psicotrópicos atuam diretamente no Sistema Nervoso Central (SNC) agindo nas ações comportamentais e de humor. A eficácia desses estímulos proporciona uma sensação de bem estar aumentando assim a procura por este tipo de fármaco (BERTRAM, 2014).

Segundo a Organização Mundial de Saúde (OMS) uma grande parte da população faz o uso de maneira incorreta o seu medicamento, acarretando com isso possíveis intoxicações. Devido ao uso abusivo e indiscriminado de determinados medicamentos, foi editada e publicada a RDC no 27 de 30 de março de 2007, que criou o Sistema Nacional para Gerenciamento de Produtos Controlados (SNGPC) (BRASIL, 2007).

O SNGPC vem com a finalidade de monitora a dispensação de medicamentos e substâncias entorpecentes e psicotrópicas e seus precursores, facilitando assim perceber fraude em qualquer processo de produção e prescrição do medicamento, permitindo o monitoramento de hábitos de prescrição e consumo de substâncias controladas em determinada região, pra propor políticas de controle (BRASIL, 2007).

O presente trabalho tem como objetivo avaliar as prescrições médicas de medicamentos de controle especial de receituário azul $\left(\mathrm{B} 1\right.$ e B2) regulamentados pela Portaria Federal $\mathrm{n}^{\circ}$ 344/98 dispensadas em uma farmácia privada do município de Poções, Bahia.

\section{Metodologia}

A presente pesquisa trata-se de um estudo exploratório descritivo quantitativo,realizada em uma drogaria, localizada na cidade de Poções - BA.

Os dados da pesquisa foram coletados após a obtenção do termo de autorização, emitido pelo responsável da drogaria e aprovação pelo Comitê de Ética em Pesquisa da FAINOR, mantendo-se o sigilo referente às informações obtidas. Para o levantamento dos dados foram avaliadas as receitasde medicamentos do receituário azul (B1 e B2)de controle especial, regulados pela Portaria Federal 344/98, dispensadas no segundo trimestre do ano de 2017. Foi seguindo o mapa do segundo trimestredo ano de 2017 que foi enviado e conferido pela Vigilância Sanitária do município. 
Cada receita que foi avaliada recebeu um número, algarismos arábicos, em ordem crescente, sendo que foi anotado respectivamente em cada formulário para possibilitar a rastreabilidade da receita caso alguma informação for esquecida de ser anotada.

As análises foram feitas com base nos parâmetros estabelecidos pela Portaria Federal 344 de 12 de maio de 1989, através de um formulário de avaliação elaborado para este fim contendo algumas questões, que serão avaliadas a legibilidade dos dados preenchidos de forma manuscrita, identificação do emitente (formulário do médico ou instituição constando nome, endereço, CRM), identificação do usuário (nome, endereço completos), quantidade em arábico e por extenso (sem emenda ou rasura) e forma farmacêutica, dosagem e posologia, quantidade aviada, data da emissão, se a dispensação estava dentro do prazo previsto por lei (30 dias), assinatura segundo a legislação que define, em caso de instituições que estavam devidamente identificadas, que o médico deverá apor sua assinatura e mais o CRM via carimbo ou manual.

Posteriormente a coleta, com os dados obtidos na pesquisa todos foram analisados e processados em programa Microsoft Excel ${ }^{\circledR} 2007$ e apresentados na forma de tabelas e gráficos.

O projeto de pesquisa foi aprovado pelo Comitê de Ética e Pesquisa (CEP) da instituição de ensino com o protocolo CAAE $\mathrm{n}^{\mathrm{o}}$ 18710813.0.0000.5578.

\section{Resultados e Discussão}

Os dados apresentados representam os receituários de medicamentos controlados que foram comercializados no segundo trimestre do ano de 2017, meses de abril a junho. Foram analisadas 600 receitas, correspondentes às prescrições médicas de medicamentos do receituário azul (B1 e B2) regulamentados pela Portaria Federal 344/1998. Pela a analise realizada 565 receitas, $94 \%$ estavam legíveis,apenas 35 receitas, $6 \%$ estavam prescritas de forma ilegível. (Gráfico 1). 
Gráfico 1. Quantitativo de receituário analisado no segundo trimestre do ano de 2017

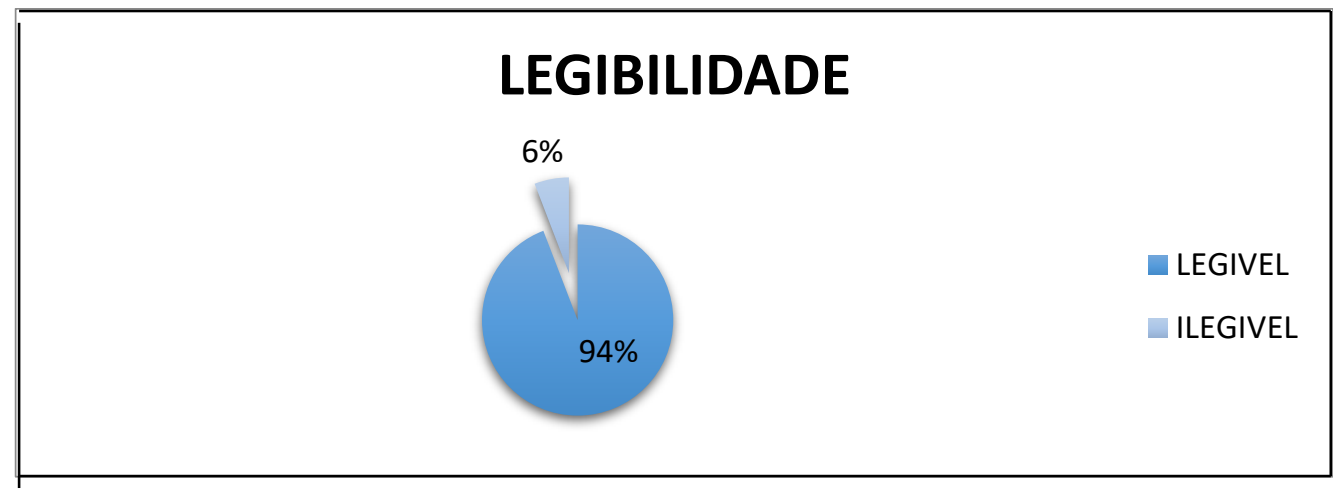

Fonte: Dados direto. Debastiani (2017).

Para que um medicamento controlado seja prescrito é necessário que o profissional prescritor tenha muita uma atenção maior no momento de elaborar o receituário. Erro como troca de medicamento por não compreender a escrita do médico, alterar a via de apresentação e dosagem são alguns erros que podem ocorrer no momento da dispensação (PAZIN FILHO et al., 2013). Esses tipos de erros podem ocasionar danos que podem ser classificados como leves ou até mesmos mais graves levando o paciente ao óbito.

Em todas as 600 prescrições tinha a identificação do emitente, ou seja, nome do medico, endereço e o número do Conselho Regional de Medicina, CRM. Quanto ao gênero dos usuários a prevalência era do sexo feminino com 427 que representa $71 \%$ do total de receitas analisadas e173 receitas, $29 \%$ foram do sexo masculino. O nome do paciente estava presente em todas as receitas analisadas, já o endereço do paciente preenchido de forma correta estava em apenas 15 receitas, $2,5 \%$, em 14 receitas 2,33\% tinha apenas o nome da cidade em que o paciente residia. Em 571 receitas que equivale a 95,16\% esses dados estava em branco, sendo esse resultado semelhante ao encontrado no estudo realizado por Nascimento et al.(2015), no qual 91,7\% das receita não apresentava o endereço do paciente.

Diante da análise realizada foi verificado que os campos da receita em que o profissional farmacêutico é responsável tais como, a quantidade dispensada, número do lote do medicamento, assinatura do farmacêutico, prazo para dispensação, estavam todas preenchida de forma correta. 
Foi observado na pesquisa que todos os receituários apresentavam a numeração de controle isso demonstra que estavam de forma correta evitando assim que houvesse qualquer tipo de fraude no documento. Quanto aos campos de informação do receituário foi verificado que em 202 receitas estavam sem a data da prescrição que representa 34\%da amostra.

$\mathrm{Na}$ tabela 1 demonstra que em 95 receitas analisadas, ou seja, 15,83\% da amostra estavam sem o campo da posologia. Para uma farmacoterapêutica correta é imprescindível que o campo posologia esteja correto de acordo com o tratamento médico, pois a sua ausência pode acarretar duvidas em relação à terapia medicamentosa. Esse dado é de grande relevância para que o paciente possa ter um tratamento correto etenha o efeito desejado pelo medicamento.

Tabela 1: Informações contidas nos receituários analisados e os dados sobre o medicamento preenchido pelo médico na receita.

\begin{tabular}{|c|c|c|c|c|}
\hline Dados sobre o medicamento & $\begin{array}{l}\text { Quantidade } \\
\text { de receitas } \\
\text { com os dados }\end{array}$ & Percentual\% & $\begin{array}{c}\text { Quantidade } \\
\text { de receitas } \\
\text { sem os dados }\end{array}$ & Percentual\% \\
\hline Data da prescrição & 398 & 66 & 202 & 34 \\
\hline Forma Farmacêutica & 600 & 100 & 0 & 0 \\
\hline Posologia & 505 & 84,16 & 95 & 15,83 \\
\hline Quantidade de medicamento & 600 & 100 & 0 & 0 \\
\hline Dosagem ou Concentração & 598 & 99,66 & 2 & 0,33 \\
\hline
\end{tabular}

Fonte: Dados direto. Debastiani (2017).

A presente pesquisa demonstra a importância de observar os dados relativos aos aspectos legais relacionados à receita médica, a começar pela forma em que foi prescrita, de forma clara até a sua dispensação pelo farmacêutico na etapa final do processo da aquisição do medicamento. O farmacêutico tem como responsabilidade orientar sobre o uso correto do medicamento ao paciente melhorando o atendimento e o direto de informação ao paciente, garantindo uma segurança do paciente levando a adesão do tratamento farmacológico (ARRAIS, 2007).

Nos dados coletados nos receituários analisados foi verificado que dentre as especialidades médicas que mais prescrevem medicamentos regulados pela Portaria Federal $344 / 98$ é Clinica geral com 53,33\% seguida por Psiquiatria com 20\% e Neurologista com 13,33\%. (Tabela 2). 
Tabela 2: Especialidade Medica que mais prescreveu benzodiazepínicos no segundo trimestre no ano de 2017

\begin{tabular}{lcc}
\hline \multicolumn{1}{c}{ Especialidades dos profissionaisprescritores } & N & \% \\
\hline Cardiologista & 59 & 9,83 \\
Clinico Geral & 320 & 53,33 \\
Nefrologista & 12 & 2 \\
Neurologista & 80 & 13,33 \\
Psiquiatria & 120 & 20 \\
Outros* (* Pediatra e Reumatologista) & 9 & 1,5 \\
\hline
\end{tabular}

Fonte: Dados direto. Debastiani (2017).

O clínico geral apesar de não possuir a especialidade para diagnosticar um transtorno mental se tornou o profissional mais presente em saúde mental no Brasil, tornando em parte, o consumo indiscriminado de fármacos de controle especial. (COSTA, 2017).

Um dos grandes problemas no país é o uso irracional de medicamentos que gera um grande desperdício de recursos e pode alterar de maneira negativa a qualidade de vida do paciente (CHAVES; JALES, 2014).

Ainda que seja obvio as vantagens da utilização dos psicofármacos em relação à qualidade terapêutica é necessário que se questione o intuito de sua utilização (BORGES, 2015). A utilização dos medicamentos classificados como psicotrópicos devem ser constantemente monitorados e avaliados.

A tabela 3 demonstra a classe e os medicamentos psicotrópicos mais prescritos no campo de pesquisa. Diante disto pode se verificar que dentro da classe dos benzodiazepínicos osmedicamentos mais prescritos foramDiazepam (25,5\%), Alprazolam (22\%) e Fenobarbital $(14,83 \%)$.

Tabela 3: Medicamentos mais prescritos no segundo trimestre do ano de 2017

\begin{tabular}{lccc}
\hline & Classe dos medicamentos & N & \% \\
\hline & Benzodiazepinas & & \\
Alprazolam & 132 & 13,5 \\
Bromazepam & 81 & 8,5 \\
Clonazepam & 51 & 9,5
\end{tabular}


Fonte: Dados direto. Debastiani (2017).

Devido ao potencial risco de dependência de psicotrópicos se faz necessário que seja feita de forma controlada desde a sua produção até parte final sendo a dispensação, tornandose indispensável à receita médica (SOUZA, 2014). No momento da dispensação é possível detectar falhas que foram cometidas no momento da prescrição diminuindo assim possíveis erros durante a administração dos medicamentos possibilitando uma melhor terapêutica para o paciente e a adesão ao tratamento prescrito.

\section{Conclusão}

A partir da análise demonstra 585 receitas não estavam preenchidas de forma correta de acordo a legislação. Isso demonstra que é necessário que para segurança do paciente os profissionais prescritores na consulta e no momento de elaborar o receituário inclua todos os dados necessários de acordo com a Portaria Federal 344/1998.

Faz-se necessário que o profissional prescritor tenha uma maior atenção no momento da prescrição, pois a falta de algumas informações na receita pode gerar problemas, dificuldade de adesão ao tratamento e até mesmo prejuízos ao paciente.

O Farmacêutico é o profissional da área responsável por todo ciclo do medicamento desde a sua produção até a sua dispensação através da analise de um receituário médico. Para que o medicamento seja dispensado de maneira correta é necessário que todos os itens dos receituários estejam descritos corretamente e legíveis.

Os dados obtidos pela pesquisa apresentam que o profissional de saúde prescritor e o Farmacêutico contribuem de maneira significativa para o Uso Racional de Medicamentos, URM dos pacientes.

Foi satisfatório demonstrar que 505 receituários, $84,16 \%$ dos dados obtidos na pesquisa no segundo trimestre do ano de 2017 apresentam os dados necessários para a dispensação correta dos medicamentos. 
A adoção de medidas básicas como realizar trabalhos educativos através da orientação e da fiscalização dos CRM cobrando que os profissionais prescritores digitalizem as prescrições, viabilizando a impressão de receitas completa, são uma das ações que poderia minimizar possíveis riscos causados num receituário ilegível que leva a uma interpretação errada provocando assim uma dispensação incorreta do medicamento.

\section{Referências}

ALBUQUERQUE, C. N de. Dicionário de termos farmacêuticos. Rio de Janeiro: Guanabara Koogan, 2009.

ARRAIS, P. S. D; BARRETO, M. L.; COELHO, H. L. L. Aspectos dos processos de prescrição e dispensação de medicamentos na percepção do paciente: estudo de base populacional em Fortaleza, Ceará, Brasil, 2007.

BERTRAM, G. K.; MASTERS, S.B.; TREVOR, A.J.; Farmacologia básica e clínica. 12.ed. - Porto Alegre: AMGH, 2014. 359p.

BORGES, Tatiana Longo et al . Prevalência do uso de psicotrópicos e fatores associados na atenção primária à saúde.Actapaul. enferm., São Paulo, v. 28, n. 4, p. 344-349, Aug. 2015. Available from. http://www.scielo.br/scielo.php?script=sci_arttext\&pid=S01031002015000400009\&lng=en\& nrm=iso $>$.access on 18 May 2017. http://dx.doi.org/10.1590/1982-0194201500058.

BRASIL. Ministério da Saúde. Cuidado farmacêutico na atenção básica:Capacitação para implantação dos serviços de clínica farmacêutica. Brasília: Ministério da Saúde, 2014. v. 2. 304 p.

Ministério da Saúde. Lei Federal No 5.991, DE 17 DE DEZEMBRO DE 1973. Dispõe sobre o controle sanitário do comércio de drogas, medicamentos, insumos farmacêuticos e correlatos, e dá outras providências. Brasília. Disponivel em: http://www.planalto.gov.br/ccivil_03/leis/L5991.htm. Acesso: 05 de maio de 2017

Ministério da Saúde. Portaria Federal No 344, DE 12 DE MAIO DE 1998. Aprova o Regulamento Técnico sobre substâncias e medicamentos sujeitos a controle especial. Diário Oficial da União, $1998 . \quad$ Disponivle http://www.anvisa.gov.br/scriptsweb/anvisalegis/VisualizaDocumento.asp?ID=939\&Versao= 2. Acesso em: 05 de maio de 2017.

Ministério da Saúde. Portaria Federal No 3916, DE 30 DE OUTUBRO DE 1998.

Politica Nacional de Medicamentos. Disponível em:


http://bvsms.saude.gov.br/bvs/saudelegis/gm/1998/prt3916_30_10_1998.html. Acesso em: 13 de stembro de 2017.

. Ministério da Saúde. Secretaria de Ciência, Tecnologia e Insumos estratégicos. Departamento de Assistência Farmacêutica e Insumos Estratégicos. Brasília, 2007. 25p.

CHAVES, M. E. T; JALES, S. T. L. Uso racional de medicamentos: uma abordagem da prescrição à dispensação. João Pessoa: EDUEPB, 2014.

COSTA, G M. P.; OLIVEIRA, M. A. S. Estudo das prescrições de psicotrópicos em uma farmácia da cidade de Sobral, Ceará, Brasil, v.29, 2017.

MOREIRA, M. P.; PINTO, T. J. A. Práticas promocionais em medicamentos sob prescrição médica: cenário e perspectivas da regulamentação brasileira e global. São Paulo, 2010

NASCIMENTO, B. R. do. Irregularidades encontradas no preenchimento de campos obrigatórios em receituários de controle especial em uma farmácia escola de viçosa, Minas Gerais. Revista Científica Univiçosa - Volume 7 - n. 1 - Viçosa - MG - Jan. - dez. 2015 - p. 5258 .

PAZIN FILHO, A. ET AL. Princípios de prescrição médica hospitalar para estudantes de medicina. Medicina, Ribeirão Preto, v.6, 2013.

SOUZA, L. M. G.Estudo de prescrições médicas de psicotrópicos de uma farmácia comercial no município de Santa Inês, maranhão, Brasil, FEBAC, 2014.

Como citar este artigo (Formato ABNT):

DEBASTIANI, Ana K.de S.; COQUEIRO, Jaqueline F.R. Análise de Prescrições Médicas de medicamentos regulados pela Portaria federal 344/1998, dispensados em uma drogaria no interior da Bahia. Id on Line Revista ultidisciplinar e de Psicologia, 2017, vol.12, n.39, p.118-127. ISSN: 19811179 .

Recebido: 15.11 .2017

Aceito: 18.11 .2017 